\title{
Disposable device offers one-stop circumcision
}

Paediatric or adult male circumcision is a common procedure worldwide, performed to address conditions such as phimosis, or to reduce the risks of penile cancer and transmission of HIV. A surgical device tested for adult circumcision-the Circular Cutter with Stapled Anastomosis for Circumcision (CCSAC), which combines foreskin removal with stapled anastomosis of inner and outer foreskin layers-compares favourably with existing methods.

\section{The CCSAC was not associated with ... oedema, infection, painful intercourse or erection difficulties 77}

A range of options are available for circumcision, from ritual tools, through basic surgery, to a number of devices that either aim to present the prepuce for surgical removal, or that use compression to cut off the blood supply to the prepuce, enabling nonsurgical removal by necrosis. Although circumcision is a simple, low-risk operation, products introduced in recent years have sought to improve safety, operational and recovery times, and cosmesis, while minimizing training requirements and costs.

The CCSAC is a single use, disposable device, invented in 2009. Following local anaesthesia, the operator stretches the prepuce over a bell-shaped shield, fixes it in place, and attaches the body of the instrument. The operator squeezes the handles of the CCSAC for $20 \mathrm{~s}$, simultaneously activating a circular cutter and inserting 18 stainless steel staples into the cut foreskin. This action releases the CCSAC from the penis, and the operator completes haemostasis by applying manual pressure for $5 \mathrm{~min}$.

In 62 patients, mean procedural time was $7.7 \pm 2.6 \mathrm{~min}$ and staples fell out from healed circumcisions within 2 weeks (10.4 \pm 1.6 days). The CCSAC was not associated with postprocedural oedema, infection, painful intercourse or erection difficulties. Patients were satisfied with the cosmetic results and would recommend use of the CCSAC to a friend.

Whether the CCSAC, with a unit cost of about US\$200, can compete in an increasingly crowded marketplace remains to be seen. Simple, disposable, plastic, compression-based devices, such as the Shang ring, PrePex and Alisklamp, provide suture-free circumcisions (in the case of the PrePex, without local anaesthesia), although some part of the device must remain in place throughout the necrosis period. Any mooted benefits of the CCSAC in treatment time, healing time and cosmesis must now be demonstrated in a broad range of patients.

Robert Phillips

Original article Yuan, Y. et al. Clinical investigation of a novel surgical device for circumcision. J. Urol. doi:10.1016/j.juro.2013.11.111 